\title{
Role of Human Liver Microsomal CYP3A4 and CYP2B6 in Catalyzing N-Dechloroethylation of Cyclophosphamide and Ifosfamide
}

\author{
Zeqi Huang, Partha Roy and David J. Waxman* \\ Division of Cell and Molecular Biology, Department of Biology, Boston University, Boston, MA \\ 02215, U.S.A.
}

\begin{abstract}
The anticancer alkylating agents cyclophosphamide (CPA) and ifosfamide (IFA) are prodrugs that undergo extensive P450-catalyzed metabolism to yield both active (4-hydroxylated) and therapeutically inactive but neurotoxic ( $\mathrm{N}$-dechloroethylated) metabolites. Whereas the human liver microsomal P450 catalysts of CPA and IFA 4-hydroxylation are well characterized, the P450 enzyme catalysts of the alternative $N$-dechloroethylation pathway are poorly defined. Analysis of a panel of fifteen human P450 cDNAs in the baculovirus expression system ('Supersomes') demonstrated that CYP3A4 exhibited the highest N-dechloroethylation activity toward both CPA and IFA, whereas CYP2B6 displayed high $\mathrm{N}$-dechloroethylation activity toward IFA, but not CPA. The contributions of each human P450 to overall liver microsomal $\mathrm{N}$ dechloroethylation were calculated using a recently described relative substrate-activity factor method, and were found to be in excellent agreement with the results of inhibition studies using the CYP3A inhibitor troleandomycin and an inhibitory monoclonal antibody to CYP2B6. With CPA as substrate, CYP3A4 was shown to catalyze $\geq 95 \%$ of liver microsomal $N$-dechloroethylation, whereas with IFA as substrate, CYP3A4 catalyzed an average of $\sim 70 \%$ of liver microsomal $N$-dechloroethylation (range $=40-90 \%$ ), with the balance of this activity catalyzed by CYP2B6 (range $=10-70 \%$, dependent on the CYP2B6 content of the liver). Because CYP2B6 can make a significant contribution to human liver microsomal IFA $N$-dechloroethylation, but only a minor contribution to IFA 4-hydroxylation, the selective inhibition of hepatic CYP2B6 activity in individuals with a high hepatic CYP2B6 content may provide a useful approach to minimize the formation of therapeutically inactive but toxic N-dechloroethylated IFA metabolites. BIOCHEM PHARMACOL 59;8:961-972, 2000. (C) 2000 Elsevier Science Inc.
\end{abstract}

KEY WORDS. ifosfamide N-dechloroethylation; cyclophosphamide; anticancer drug metabolism; CYP3A4; CYP2B6; oxazaphosphorine metabolism

$\mathrm{CPA} \dagger$ and its structural isomer, IFA, are oxazaphosphorine alkylating agents that are widely used in cancer chemotherapy and for immunosuppression [1]. CPA and IFA are both prodrugs that can be metabolized via two alternative CYP-catalyzed pathways, 4-hydroxylation and N-dechloroethylation [2]. 4-Hydroxylation corresponds to a drug activation pathway that yields an active alkylating species (phosphoramide mustard or isophosphoramide mustard) and equimolar amounts of the toxic by-product acrolein. Acrolein is responsible for urotoxicity, primarily hemorrhagic cystitis, which can be minimized by treatment of patients with mesna, a uroprotective agent [3]. N-Dechloroethylation generates inactive oxazaphosphorine metabolites (2- and 3-dechloroethyl-IFA, in the case of IFA), and

\footnotetext{
* Corresponding author: David J. Waxman, Ph.D., Department of Biology, Boston University, 5 Cummington St., Boston, MA 02215. Tel. (617) 353-7401; FAX (617) 353-7404; E-mail: djw@bio.bu.edu

$\dagger$ Abbreviations: CPA, cyclophosphamide; IFA, ifosfamide; CYP, cytochrome P450; HLM, human liver microsomes; Mab2B6, monoclonal antibody to CYP2B6; TAO, troleandomycin; RSF, relative substrateactivity factor; and CA, chloroacetaldehyde.

Received 27 May 1999; accepted 20 September 1999.
}

a toxic by-product, CA. CA can cause damage to proximal tubules and to neurons, and is largely responsible for the nephrotoxicity [4-6] and neurotoxicity [7, 8] associated with IFA treatment.

Despite their structural similarities, CPA and IFA exhibit important differences in their metabolism, toxicity, and therapeutic spectrum. About $45 \%$ of a therapeutic dose of IFA is typically metabolized via $\mathrm{N}$-dechloroethylation to CA, whereas only $10 \%$ of CPA is converted to CA [9]. Consequently, many more patients develop CA-induced neurotoxicity and nephrotoxicity after IFA administration than after CPA treatment. Clinically, neurotoxicity and nephrotoxicity are important dose-limiting factors for IFA treatment $[1,10]$. Neurotoxicity occurs in about $20 \%$ of patients after IFA administration, but is rare in CPAtreated patients. IFA, however, has lower myelotoxicity than CPA, which makes it possible to use higher doses of IFA [9]. For these reasons, it is likely that the safety and perhaps the therapeutic effects of IFA could be improved if ways could be found to minimize CA formation via the P450-catalyzed $\mathrm{N}$-dechloroethylation pathway.

A definitive identification of the individual human P450 
enzyme catalysts of the 4-hydroxylation and $\mathrm{N}$-dechloroethylation of CPA and IFA is needed to develop effective approaches to selectively minimize CA formation. The 4-hydroxylation pathway has been studied extensively due to the availability of sensitive methods to detect acrolein, a metabolite of the 4-hydroxylation pathway that can easily be measured fluorometrically by condensation with 3-aminophenol to yield 7-hydroxyquinoline [11-14], and the P450 catalysts of this reaction are well characterized [15-20]. The N-dechloroethylation of CPA and IFA has not been well studied because of a lack of sensitive methods. Recently, methods have been developed to determine dechloroethyl-IFA [21] and CA [22, 23], the metabolic products of IFA $\mathrm{N}$-dechloroethylation. IFA $\mathrm{N}$-dechloroethylation has been investigated using rat liver microsomes [24, 25] and human liver microsomes [19, 26]. In the rat, both CYP3A and CYP2B enzymes are involved in catalyzing IFA N-dechloroethylation [24]. CYP3A4 is reported to be the only human P450 enzyme that catalyzes this reaction [19, 26]; however, only a limited subset of human P450s was examined. A more definitive evaluation of the human P450s that contribute to liver microsomal IFA $\mathrm{N}$-dechloroethylation is clearly required if effective approaches to modulate this reaction in cancer patients are to be identified.

In this study, we applied a newly developed HPLCfluorometric method for CPA and IFA $\mathrm{N}$-dechloroethylation [27] to identify the human P450 enzymes that catalyze this reaction in human liver microsomes and as cDNAexpressed enzymes. These studies used P450 form-selective chemical and antibody inhibitors and produced results in excellent agreement with those determined using an RSF method [15] to calculate P450 form-specific contributions to overall liver microsomal metabolism based on the measured P450 enzyme content of each liver sample and the specific catalytic activity of the expressed P450s. Both CYP2B6 and CYP3A4 were shown to make important contributions to IFA $N$-dechloroethylation in human liver microsomes. These findings are discussed in the context of using CYP2B6 form-selective inhibitors to minimize the undesirable $\mathrm{N}$-dechloroethylation of IFA.

\section{MATERIALS AND METHODS}

\section{Chemicals}

Adenosine, TAO, CPA, and NADPH were purchased from the Sigma Chemical Co. CA $(50 \%, w / v$, in water) was purchased from the Aldrich Chemical Co. IFA was obtained from the Drug Synthesis and Chemistry Branch of the National Cancer Institute. 4-Hydroperoxy-CPA and 4-hydroperoxy-IFA were gifts from Dr. J. Pohl (ASTA Pharma). Methanol (HPLC grade) was purchased from J. T. Baker.

\section{Enzymes}

Human liver microsomes were those used previously (liver samples HLS2, HLS8, HLS9, and HLS25) [15] or were purchased from the Gentest Corp., with donor information and P450 form-diagnostic substrate activities for each of the major human liver microsomal $\mathrm{P} 450$ s provided by the supplier. Human liver microsome sample H161 (Gentest Corp.) used in this study corresponds to a pool of microsomes from six human livers: livers H003, H023, H051, H086 (20\% each) and livers H030 and H043 (10\% each) (see Table 1, below). Supersomes expressing individual human P450 cDNAs, and the inhibitory monoclonal antibody to CYP2B6 [28], were obtained from the Gentest Corp. Supersomes are microsomes prepared from baculovirus-infected insect cells that express single human P450s, human NADPH P450 reductase, and in some cases, cytochrome $b_{5}$. Supersomes containing $\mathrm{P} 450$ reductase and cytochrome $b_{5}$ but no cytochrome $\mathrm{P} 450$ were used as negative controls in each series of enzyme assays.

\section{In Vitro Microsomal Assays}

The P450 metabolites of CPA and IFA, CA (formed by the $N$-dechloroethylation pathway) and acrolein (formed by the 4-hydroxylation pathway), were determined in parallel using a single incubation tube [27]. Incubations were carried out for $30 \mathrm{~min}$ in a total volume of $100 \mu \mathrm{L}$, and included $50 \mathrm{mM}$ potassium phosphate buffer, $\mathrm{pH}$ 7.4, 0.1 mM EDTA, 1 mM NADPH, 10 pmol Supersomes P450 or $100 \mu \mathrm{g}$ of human liver microsomes, and $0.25 \mathrm{mM}$ CPA or IFA as substrate. Product formation was linear with time and enzyme concentration under these assay conditions. Eight substrate concentrations $[0,0.15,0.25,0.5,1,2,4$, and $8 \mathrm{mM}$ ] were used for enzyme kinetic analyses. Reaction mixtures were preincubated in a $37^{\circ}$ water bath for $4 \mathrm{~min}$ prior to the addition of NADPH. Reactions were initiated by adding NADPH, and then the mixture was incubated for $30 \mathrm{~min}$ in a $37^{\circ}$ water bath with gentle shaking. After incubation, $40 \mu \mathrm{L}$ of ice-cold $5.5 \% \mathrm{ZnSO}_{4}, 40 \mu \mathrm{L}$ of saturated barium hydroxide, and $20 \mu \mathrm{L}$ of $0.01 \mathrm{~N} \mathrm{HCl}$ were added sequentially to each tube, followed by centrifugation in a microfuge at $14,000 \mathrm{rpm}$ for $20 \mathrm{~min}$ to precipitate the protein. The supernatants were divided into two $80-\mu \mathrm{L}$ aliquots, which were used to measure CA and acrolein, respectively. For CA measurement, $10 \mu \mathrm{L}$ of $2 \mathrm{M}$ sodium acetate, $\mathrm{pH} 4.5$, and $10 \mu \mathrm{L}$ of $100 \mathrm{mM}$ adenosine were added to $80 \mu \mathrm{L}$ of supernatant and heated at $80^{\circ}$ for $2 \mathrm{hr}$ to form 1-N ${ }^{6}$-ethenoadenosine [27]. To assay for acrolein, 45 $\mu \mathrm{L}$ of a fluorescent mixture $(6 \mathrm{mg} / \mathrm{mL}$ of 3 -aminophenol and $6 \mathrm{mg} / \mathrm{mL}$ of hydroxylamine hydrochloride freshly dissolved in $1 \mathrm{~N} \mathrm{HCl}$ ) was added to $80 \mu \mathrm{L}$ of supernatant, and then the samples were heated at $90^{\circ}$ for $30 \mathrm{~min}$ to form 7 -hydroxyquinoline $[12,13]$. Samples were protected from light during and after derivatization. The derivatized samples were injected directly onto a $\mathrm{C}_{18}$ HPLC column for analysis as described below.

\section{HPLC}

The components of the Waters HPLC system (Waters Corp.) are detailed elsewhere [27]. Samples were chromato- 
TABLE 1. 4-Hydroxylation and N-dechloroethylation of CPA and IFA assayed in a panel of human liver microsomes

\begin{tabular}{|c|c|c|c|c|c|c|}
\hline HLM* & \multicolumn{2}{|c|}{$\mathrm{CPA}$} & \multicolumn{2}{|c|}{ IFA } & \multicolumn{2}{|c|}{ Ratio (CPA/IFA) } \\
\hline $\mathrm{H} 003$ & 0.199 & 15 & 0.165 & 41 & 1.76 & 0.43 \\
\hline $\mathrm{H} 023$ & 0.082 & 12 & 0.049 & 35 & 2.29 & 0.55 \\
\hline H030 & 0.249 & 20 & 0.228 & 40 & 1.47 & 0.53 \\
\hline $\mathrm{H} 042$ & 0.618 & 19 & 0.645 & 55 & 1.71 & 0.33 \\
\hline H066 & 0.198 & 22 & 0.217 & 50 & 1.42 & 0.40 \\
\hline H070 & 0.260 & 34 & 0.333 & 41 & 0.87 & 0.65 \\
\hline H089 & 0.274 & 28 & 0.282 & 48 & 1.34 & 0.57 \\
\hline H093 & 0.097 & 24 & 0.108 & 37 & 1.08 & 0.58 \\
\hline $\mathrm{H} 112$ & 0.637 & 30 & 0.721 & 54 & 1.36 & 0.48 \\
\hline H161 & 0.328 & 14 & 0.299 & 50 & 1.88 & 0.31 \\
\hline Mean & 0.284 & 19 & 0.276 & 39 & 1.47 & 0.48 \\
\hline Range & $0.075-0.669$ & $4-34$ & $0.049-0.721$ & $8-55$ & $0.84-2.58$ & $0.16-0.58$ \\
\hline
\end{tabular}

*Human microsomes were from a Gentest panel (livers H003-H161) or from a liver panel described previously (HLS2-HLS25) [15].

† Sum of microsomal 4-hydroxylation and $\mathrm{N}$-dechloroethylation, measured at $0.25 \mathrm{mM} \mathrm{CPA}$ or $0.25 \mathrm{mM}$ IFA and expressed as nmol total product formed/min/mg of liver microsomal protein.

$\$$ Percentage of liver microsomal metabolism proceeding via the $N$-dechloroethylation pathway.

graphed on a Luna $\mathrm{C}_{18}$ (2) column (Phenomenex), which is stable under the acidic conditions required for analysis of 7-hydroxyquinoline fluorescence. For CA analysis, the $\mathrm{C}_{18}$ column was eluted with $20 \%$ methanol in water and run isocratically at a flow rate of $1 \mathrm{~mL} / \mathrm{min}$. The fluorescent product $1-\mathrm{N}^{6}$-ethenoadenosine eluted at $5.7 \mathrm{~min}$ and was monitored for fluorescence by excitation at $270 \mathrm{~nm}$ and emission at $411 \mathrm{~nm}$ with a gain of 10 and a slit setting of 18 . To assay for acrolein, the column was eluted with $20 \%$ methanol in $0.5 \% \mathrm{H}_{3} \mathrm{PO}_{4}$ (pH $\sim 1.8$ ) isocratically at a flow rate of $1 \mathrm{~mL} / \mathrm{min}$. The fluorescent product 7 -hydroxyquinoline eluted at $3.2 \mathrm{~min}$ and was monitored at $350 \mathrm{~nm}$ (excitation) and $515 \mathrm{~nm}$ (emission) with a gain of 10 and a slit of 18 . Quantitations were based on integrated peak areas determined using Millenium ${ }^{32}$ software (Waters Corp.) and calculated from calibration curves generated by incubation of standards in BSA under the same assay conditions [27].

\section{P450 Inhibition Studies}

For inhibition with Mab2B6, human liver microsomes were incubated with Mab2B6 on ice for $30 \mathrm{~min}$. Then substrate, assay buffer, and NADPH were added, and assays were carried out as described above. For CYP3A inhibition studies, TAO was dissolved in methanol and added to individual assay tubes as required. Next, the methanol was evaporated using a Speed-Vac concentrator. TAO was incubated with human liver microsomes for $30 \mathrm{~min}$ at $37^{\circ}$ in the presence of NADPH to allow for formation of the inactive TAO-protein complex. Then substrate (CPA or IFA at $0.25 \mathrm{mM}$ ) and a fresh aliquot of NADPH were added to the tubes, which were incubated for an additional $30 \mathrm{~min}$ at $37^{\circ}$. Finally, samples were processed as described above.

\section{RSF Calculations}

The RSF value for each specific cDNA-expressed P450 is defined as the ratio of the catalytic activity of that P450 in a test substrate reaction (e.g. IFA $N$-dechloroethylation) to its activity assayed with a diagnostic, P450 form-selective substrate [15]. The test substrate catalytic activity attributable to each $\mathrm{P} 450$ in a given human liver microsome sample was calculated by multiplying the RSF value of each P450 by the diagnostic activity measured for that $\mathrm{P} 450$ in the human liver microsome sample. Then the contribution of each P450 form to the test substrate activity in human liver microsomes was calculated as a percentage of the total calculated microsomal P450 activity. A more detailed description of the RSF method for calculation of microsomal P450 activities and the potential limitations of this approach are provided elsewhere [15].

\section{Data Analysis}

Data were managed with Microsoft Excel and presented using GraphPad Prism software (GraphPad Software, Inc.). The parameters for enzyme kinetics were determined using Enzymekinetics v.1.11 software (Trinity Software). Data shown generally are based on duplicate determinations for each data point. 

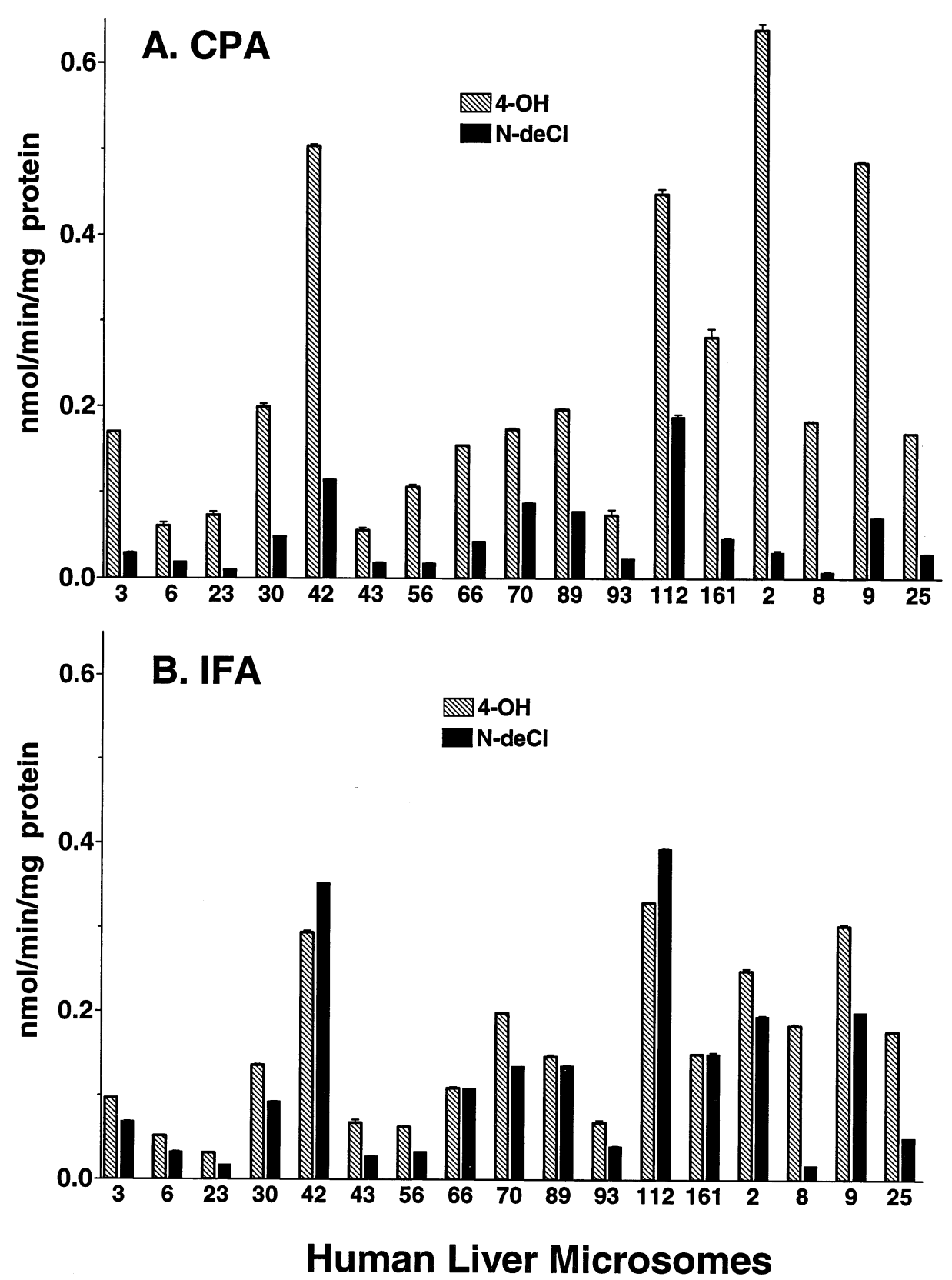

FIG. 1. Metabolism of CPA and IFA by human liver microsomes. 4-Hydroxylation and N-dechloroethylation activities were determined as described in Materials and Methods at a substrate concentration of $0.25 \mathrm{mM}$ CPA or IFA. Liver samples and activities correspond to those shown in Table 1. Sample H161 is a pool of human liver microsomes (see Materials and Methods). Data shown represent the means \pm half the range of enzyme activities based on duplicate determinations. In most cases, the error bars are too small to be visible.

\section{RESULTS}

Metabolism of CPA and IFA by Human Liver Microsomes

The 4-hydroxylation and N-dechloroethylation of CPA and IFA were assayed in a panel of seventeen human liver microsomes. Substantial variation among the individual livers with respect to total metabolism and the pattern of metabolites was apparent (Table 1, Fig. 1). Substantial differences $(>10$-fold) in the rates of microsomal CPA $\mathrm{N}$-dechloroethylation and IFA $\mathrm{N}$-dechloroethylation were observed within the panel of livers. With CPA as substrate, all of the livers showed at least 2-fold higher 4-hydroxylation than $\mathrm{N}$-dechloroethylation activity. With IFA, the two P450 metabolic pathways were catalyzed at similar rates in a majority of livers. Based on initial reaction rates, the percentage of metabolism proceeding via $\mathrm{N}$-dechloroethylation ranged from 4 to $34 \%$ (mean 19\%) for CPA and from 8 to $55 \%$ (mean 39\%) for IFA (Table 1). This finding is consistent with clinical data showing that a much larger fraction of IFA than CPA undergoes $\mathrm{N}$-dechloroethylation [10]. However, the total rates of metabolism (4-hydroxylaton $+\mathrm{N}$-dechloroethylation) for CPA and IFA were 
strikingly similar in each liver (Table 1), suggesting that the increased $N$-dechloroethylation of IFA occurs at the expense of the competing 4-hydroxylation pathway. Correlation analyses based on diagnostic, P450 form-selective activities measured for each liver in the panel revealed that CPA and IFA $N$-dechloroethylation both correlated weakly with total microsomal P450 content (CPA: $r=$ 0.5537, $P<0.05$; IFA: $r=0.5633, P<0.05$ ), and more strongly with microsomal testosterone $6 \beta$-hydroxylase activity (CYP3A-dependent) (CPA: $r=0.9391$; IFA: $r=$ 0.9093, $P<0.001)$ and $(S)$-mephenytoin $N$-demethylase activity (CYP2B6-dependent) (CPA: $r=0.6996, P<0.01$; IFA: $r=0.8827, P<0.001)$. Significant correlations were not found with other P450 form-diagnostic liver microsomal activities, including phenacetin O-deethylase (CYP1A2), coumarin 7-hydroxylase (CYP2A6), paclitaxel 6 $\alpha$-hydroxylase (CYP2C8), diclofenac 4'-hydroxylase (CYP2C9), (S)-mephenytoin 4-hydroxylase (CYP2C19), bufuralol 1'-hydroxylase (CYP2D6), chlorzoxazone 6-hydroxylase (CYP2E1), lauric acid 12-hydroxylase (CYP4A11), and methyl p-tolyl sulfide oxidase (flavin monooxygenase) (data not shown).

\section{Metabolism of CPA and IFA by Individual cDNA- Expressed Human P450s (Supersomes)}

Fifteen cDNA-expressed human P450 enzymes were assayed for CPA and IFA metabolism at $0.25 \mathrm{mM}$ substrate (Fig. 2). With CPA as substrate, CYP2B6 showed the highest 4-hydroxylase activity, followed by CYP3A4 and CYP2C19. Several other P450s, including CYPs 1A1, 2A6, 2C8, 2C9, and 3A7, exhibited low CPA 4-hydroxylase activity. CYP3A4 catalyzed a very strong CPA $\mathrm{N}$-dechloroethylation activity, followed by CYP3A7 and CYP3A5. The other twelve P450s were essentially inactive. With IFA as substrate, CYP3A4 catalyzed the highest 4-hydroxylase activity, followed by CYPs 2B6, 2C19, 2A6, and 1A1. CYP3A4 and CYP2B6 showed the highest rates of IFA $\mathrm{N}$-dechloroethylation, whereas the other $\mathrm{P} 450$ s exhibited little (CYPs 3A5, 3A7, and 2C19) or no activity in this reaction. Based on the initial rates shown in Fig. 2, the percent $N$-dechloroethylation catalyzed by CYP2B6 was $0 \%$ for CPA and $80 \%$ for IFA, whereas for CYP3A4, the percent $N$-dechloroethylation was $71 \%$ for CPA and $51 \%$ for IFA.

\section{Role of Individual P450s in IFA and CPA N. Dechloroethylation Based on the RSF Method}

We recently described an RSF method that can be used to calculate the contribution of individual P450s to human liver microsomal CPA and IFA metabolism based on cDNA-expressed P450 activities and liver microsomal P450 profiles [15]. Using the cDNA-expressed N-dechloroethylase activities (Fig. 2) and the P450 form-diagnostic substrate activities obtained from Gentest for the panel of Supersomes and the panel of human liver microsomes, the
CPA and IFA $N$-dechloroethylase activities attributable to each human liver $\mathrm{P} 450$ form were calculated. These values, in turn, were summed to obtain the total RSF-calculated microsomal CPA and IFA $N$-dechloroethylase activity for each liver in the panel. The results obtained correlated very well with the corresponding measured enzyme activities (Fig. 1) in the panel of seventeen human liver microsomes (Fig. 3, A and B), supporting the validity of the RSF approach.

The contribution of each P450 form to liver microsomal CPA and IFA $\mathrm{N}$-dechloroethylation was also determined by the RSF method (Table 2). In the average liver sample in the panel, the major fraction of CPA N-dechloroethylation activity $(\sim 96 \%)$ was determined to be catalyzed by CYP3A enzymes. CYP3A was also found to be the major P450 catalyst of IFA $N$-dechloroethylation ( $\sim 70 \%$ of total activity) by the RSF method, with the balance of the activity $(\sim 27 \%)$ catalyzed by CYP2B6 in the average liver. The contribution of the other P450s to CPA and IFA $N$-dechloroethylation was negligible (Table 2). Furthermore, as shown in Table 3, the contribution of CYP3A4 to CPA N-dechloroethylation was found to be consistently high in all human livers ( $\geq 90 \%$ in nearly all samples). By contrast, the CYP3A4 contribution to IFA $\mathrm{N}$-dechloroethylation varied from 38.8 to $87.5 \%$, while that of CYP2B6 ranged from as low as 9.8\% (liver HLS25, which has a very low CYP2B6 content) to 58.2\% (liver HLS2, which has a high CYP2B6 content).

The contribution of each hepatic P450 to CPA and IFA 4-hydroxylation was also determined by the RSF approach (Table 2). CYP2B6 and CYP3A4 were found to be the major P450s responsible for CPA 4-hydroxylation ( $\sim 45$ and $\sim 25 \%$ of total activity, respectively), while CYP3A4 was found to be the major P450 responsible for IFA 4 -hydroxylation ( $\sim 65 \%$ of total activity). CYP2B6 contributed to a minor extent $(\sim 6.5 \%)$ to microsomal IFA 4-hydroxylation. CYP1A2, CYP2A6, and CYP2C9 also contributed to CPA and IFA 4-hydroxylation to a small extent (5-12\% each), with CYP2C9 contributing to a greater extent to CPA 4-hydroxylation and CYP2A6 contributing more significantly to IFA 4-hydroxylation. These latter results are similar to those obtained by the RSF method in assays carried out at a much higher substrate concentration $(2 \mathrm{mM})$ in studies using less sensitive analytical methods [15]. Finally, total human liver microsomal 4-hydroxylation activities calculated by the RSF method were in good agreement with experimental values (Fig. 3, C and D).

\section{Inhibition of Microsomal N-Dechloroethylation by TAO and Mab2B6}

The P450 form-dependent contributions to CPA and IFA $\mathrm{N}$-dechloroethylation determined by the RSF method were tested experimentally in a pooled human liver microsome sample (H161) using TAO, a CYP3A form-selective chemical inhibitor, and Mab2B6, a monoclonal antibody inhib- 

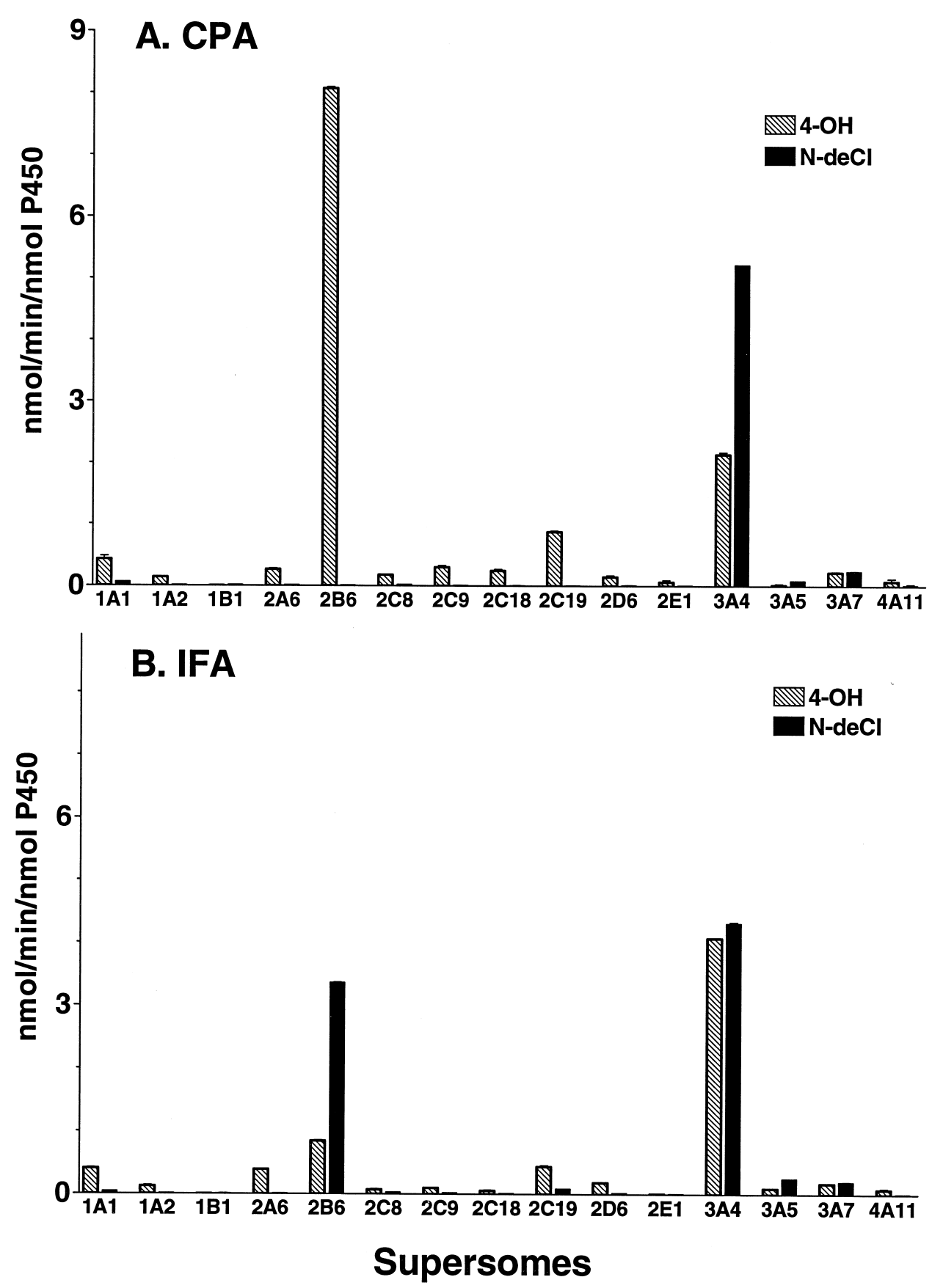

FIG. 2. Metabolism of CPA and IFA by cDNA-expressed human P450s ('Supersomes'). Activities were determined at 0.25 mM CPA or IFA as detailed in Materials and Methods. All of the Supersomes coexpressed human P450 reductase with the indicated human P450. Supersomes CYP2B6, 2C8, 2C9, 2C18, 2C19, 2E1, 3A4, 3A5, and 3A7 also contained human cytochrome $b_{5}$. CYP2C9 corresponds to the Arg144 allele, and CYP3A5 corresponds to lot E43 [15], which contains cytochrome $b_{5}$ and P450 at a molar ratio of 1.5:1. Data shown represent the means \pm half the range of enzyme activities based on duplicate determinations.

itory to CYP2B6 (Fig. 4). TAO inhibited $>90 \%$ of microsomal CPA N-dechloroethylation and $\sim 50 \%$ of microsomal IFA N-dechloroethylation (panels $\mathrm{B}$ and $\mathrm{D}$, respectively). Mab2B6 inhibited $\sim 40 \%$ of IFA $N$-dechloroethylation, with little effect on CPA $N$-dechloroethylation. These findings are consistent with the values calculated using the RSF method. Examination of the effects of these inhibitors on microsomal 4-hydroxylation activity, measured in the same incubations, revealed that TAO inhibited IFA 4-hydroxylation and IFA $N$-dechloroethylation to the same extent (Fig. 4D), consistent with the finding that CYP3A4 catalyzes these two reactions at the same rate (Fig. 2). In contrast, TAO inhibited CPA 4-hydroxylation by only 10-15\% (Fig. 4B), consistent with the low CYP3A contribution to this activity calculated for liver sample H161 (Table 3).

Livers H042 and HLS2, which are both CYP2B6-rich, and liver HLS25, a CYP2B6-poor liver, were also tested for 


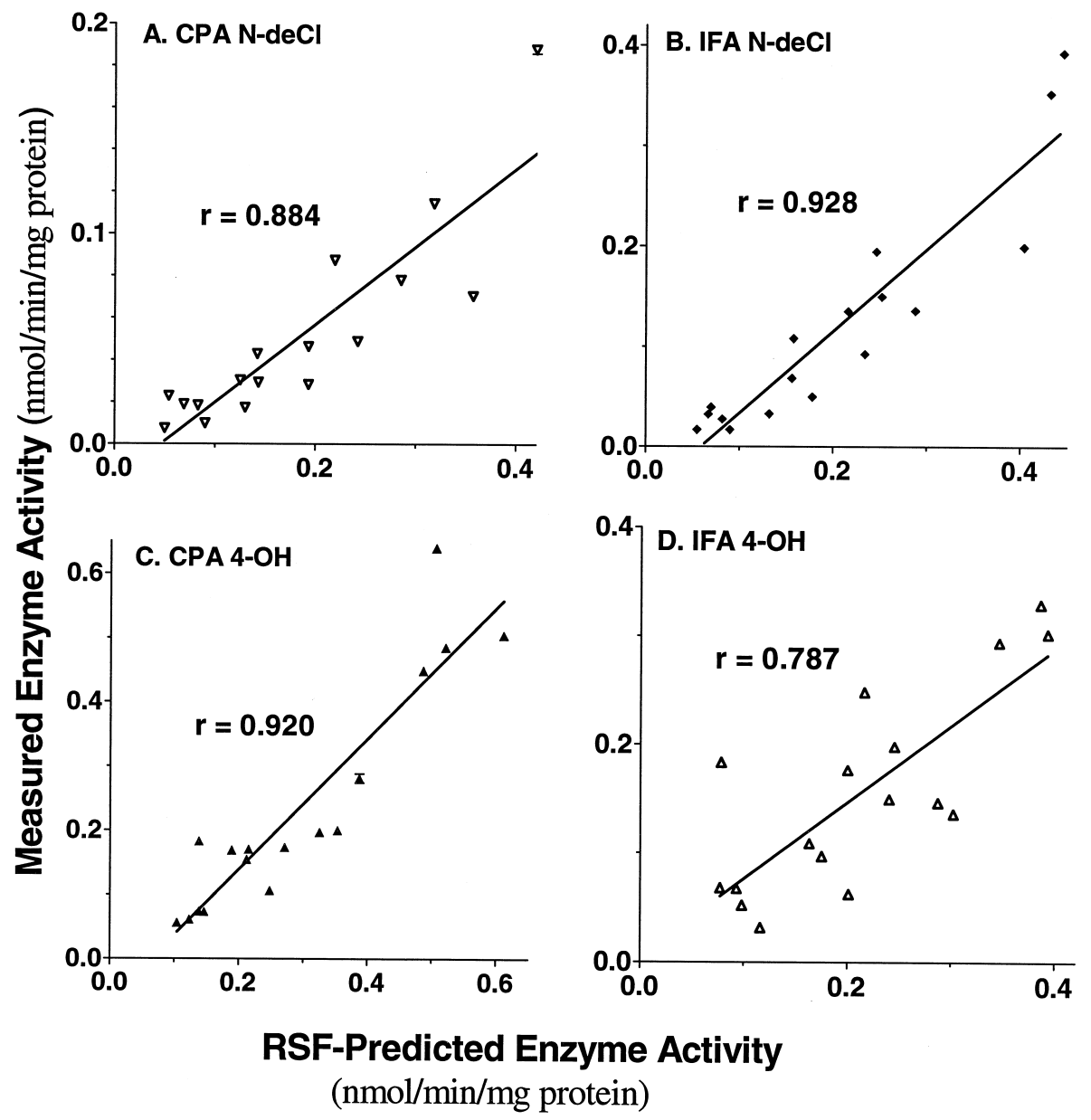

FIG. 3. Correlation between measured activities and predicted activities in a panel of microsomes from seventeen human livers. The experimental activities graphed on each $y$-axis are those shown in Fig. 1. The predicted activities graphed on each $\mathrm{x}$-axis are total liver microsomal activities calculated by the RSF method.

the inhibition of IFA $\mathrm{N}$-dechloroethylation by TAO and Mab2B6. The results obtained were in good agreement with the $\mathrm{P} 450$ form-specific contributions predicted by the RSF method (Table 4). The CYP2B6 experimental activities based on inhibition with Mab2B6 were somewhat higher

TABLE 2. Percent contribution of each individual P450 to CPA and IFA metabolism in the average human liver sample determined using the RSF method*

\begin{tabular}{lrrrrr}
\hline & \multicolumn{2}{c}{$\mathrm{N}$-deCl } & & \multicolumn{2}{c}{ 4-OH } \\
\cline { 2 - 3 } \cline { 5 - 6 } CYP & CPA & IFA & & CPA & IFA \\
\hline 1A2 & 1.2 & 0.5 & 7.0 & 8.5 \\
2A6 & 0.6 & 0.2 & 6.2 & 12.5 \\
2B6 & 0.1 & 26.5 & & 44.8 & 6.5 \\
2C8 & 0.5 & 0.5 & & 2.3 & 1.3 \\
2C9 & 0.7 & 1.2 & & 12.0 & 5.4 \\
2C19 & 0.0 & 0.1 & & 0.6 & 0.4 \\
2D6 & 0.0 & 0.0 & & 0.2 & 0.3 \\
2E1 & 0.6 & 0.4 & & 2.1 & 0.4 \\
3A4 & 96.4 & 70.6 & & 24.7 & 64.8 \\
& & & & & \\
\hline
\end{tabular}

*Values shown are the percentages of total microsomal P450 activity attributable to the indicated P450 forms. The major P450 contributors to each activity are highlighted in bold. than the values predicted by the RSF method, whereas the CYP3A4-dependent activities indicated by TAO inhibition were very similar to the RSF-predicted values.

\section{Enzyme Kinetics}

Steady-state enzyme kinetics were evaluated to further characterize the role of CYPs 2B6 and 3A4 in CPA and IFA $N$-dechloroethylation. CYP3A4 was found to be a high $K_{m}$ and high $V_{\max }$ catalyst of CPA $N$-dechloroethylation, with an overall high catalytic efficiency, as judged by the in vitro intrinsic clearance $\left(V_{\max } / K_{m}\right)$ (Table 5). CYP3A4 was also an efficient catalyst of IFA $N$-dechloroethylation, with a slightly lower $K_{m}$ and $V_{\max }$ than seen with CPA, but with a very similar in vitro intrinsic clearance. CYP2B6 exhibited a higher $K_{m}$ and a lower $V_{\max }$ than CYP3A4 for IFA $N$-dechloroethylation. Consequently, the apparent $V_{\max } / K_{m}$ of CYP2B6 for this activity was only half that of CYP3A4.

$K_{m}$ and $V_{\text {max }}$ parameters for CPA and IFA 4-hydroxylation catalyzed by CYPs $2 \mathrm{~B} 6$ and 3A4 were determined from the same incubations. In the 4-hydroxylation reaction, CYP2B6 showed a similar $K_{m}$ value for both CPA and IFA, but the $V_{\max }$ was 10 times higher with CPA as substrate 
TABLE 3. Contribution of CYP2B6 and CYP3A4 to microsomal 4-hydroxylation and N-dechloroethylation of CPA and IFA in individual human liver microsomes determined using the RSF method*

\begin{tabular}{|c|c|c|c|c|c|c|c|c|}
\hline \multirow[b]{3}{*}{$\mathrm{HLM}_{\dagger}$} & \multicolumn{4}{|c|}{ CPY2B6 } & \multicolumn{4}{|c|}{ CYP3A4 } \\
\hline & \multicolumn{2}{|c|}{ CPA } & \multicolumn{2}{|c|}{ IFA } & \multicolumn{2}{|c|}{ CPA } & \multicolumn{2}{|c|}{ IFA } \\
\hline & $4-\mathrm{OH}$ & $\mathrm{N}-\mathrm{deCl}$ & $4-\mathrm{OH}$ & $\mathrm{N}-\mathrm{deCl}$ & $4-\mathrm{OH}$ & $\mathrm{N}$-deCl & $4-\mathrm{OH}$ & $\mathrm{N}-\mathrm{deCl}$ \\
\hline H003 & 41.7 & $\leq 0.2$ & 5.4 & 24.0 & 26.4 & 96.6 & 62.0 & 73.3 \\
\hline H006 & 18.5 & $\leq 0.2$ & 2.4 & 14.2 & 21.1 & 91.8 & 50.5 & 78.3 \\
\hline H023 & 25.1 & $\leq 0.2$ & 3.3 & 16.9 & 23.7 & 94.0 & 57.0 & 77.5 \\
\hline H030 & 23.1 & $\leq 0.2$ & 2.8 & 14.5 & 26.5 & 94.6 & 59.1 & 80.6 \\
\hline $\mathrm{H} 042$ & 67.2 & $\leq 0.2$ & 12.4 & 39.5 & 20.9 & 98.0 & 70.3 & 59.4 \\
\hline H043 & 30.0 & $\leq 0.2$ & 3.5 & 15.8 & 31.8 & 96.7 & 67.7 & 81.0 \\
\hline H056 & 24.4 & $\leq 0.2$ & 3.1 & 19.1 & 19.7 & 91.4 & 46.2 & 74.5 \\
\hline H066 & 46.0 & $\leq 0.2$ & 6.2 & 25.7 & 26.6 & 97.0 & 65.6 & 72.0 \\
\hline H070 & 30.7 & $\leq 0.2$ & 3.5 & 16.0 & 32.1 & 96.9 & 67.5 & 80.9 \\
\hline H089 & 39.1 & $\leq 0.2$ & 4.6 & 18.4 & 35.0 & 97.5 & 75.5 & 79.7 \\
\hline H093 & 43.2 & $\leq 0.2$ & 8.1 & 35.5 & 14.7 & 91.4 & 49.9 & 58.4 \\
\hline H112 & 48.4 & $\leq 0.2$ & 6.4 & 21.9 & 35.0 & 98.8 & 83.9 & 76.7 \\
\hline HLS2 & 68.1 & $\leq 0.2$ & 16.7 & 58.2 & 9.4 & 92.8 & 41.9 & 38.8 \\
\hline HLS8 & 19.8 & $\leq 0.2$ & 3.7 & 20.7 & 12.8 & 86.7 & 43.1 & 64.7 \\
\hline HLS9 & 49.7 & $\leq 0.2$ & 6.9 & 26.7 & 27.5 & 97.7 & 69.3 & 71.4 \\
\hline HLS25 & 22.1 & $\leq 0.2$ & 2.2 & 9.8 & 40.8 & 97.5 & 73.3 & 87.5 \\
\hline H161 & 57.5 & $\leq 0.2$ & 9.7 & 36.8 & 19.7 & 96.4 & 60.6 & 61.0 \\
\hline Mean & 44.9 & $\leq 0.2$ & 6.4 & 26.5 & 24.7 & 96.4 & 64.8 & 70.6 \\
\hline Range & $18-68$ & $0-0.2$ & $2-17$ & $10-58$ & $9-41$ & 87-99 & $43-84$ & $39-87$ \\
\hline
\end{tabular}

than with IFA. In contrast, CYP3A4 showed a 2-fold higher $V_{\max }$ toward IFA than toward CPA, but with a similar $K_{m}$. The $K_{m}$ values for the 4-hydroxylation reactions reported here are similar to those in a previous study [15]. The $V_{\max }$ values, however, were several-fold higher than reported previously [15].

\section{DISCUSSION}

The metabolism of CPA and IFA via N-dechloroethylation, which generates the neurotoxic and nephrotoxic metabolite CA, was assayed using human liver microsomes and a panel of cDNA-expressed human P450 enzymes. The contribution of each P450 form to liver microsomal Ndechloroethylation of CPA and IFA was determined experimentally by inhibition with P450 form-specific chemical and antibody inhibitors, and by calculation, using a recently described RSF method [15] that is based on measured cDNA-expressed P450 activities and liver microsomal P450 contents. The results obtained established that CYPs 2B6 and $3 \mathrm{~A} 4$ both make significant contributions to human liver microsomal IFA $\mathrm{N}$-dechloroethylation, with the relative contribution dependent on the microsomal P450 content of each liver. In contrast, CPA N-dechloroethylation was shown to be catalyzed by CYP3A4 alone.* These studies used a highly sensitive HPLC fluorescence method

\footnotetext{
* Some contribution from the other, more minor (and less active) human liver CYP3A enzymes may also occur. The TAO inhibition and RSF calculation methods do not distinguish between the individual CYP3A enzymes.
}

recently developed in this laboratory to measure the individual P450 metabolites of CPA or IFA in a single incubation tube [27]. This method enabled us to assay the metabolism of these anticancer prodrugs more accurately and at a much lower substrate concentration $(0.25 \mathrm{mM})$ and using shorter incubation times than were possible with earlier methods. Using this method, P450 form-specific contributions to CYP and IFA 4-hydroxylation could also be identified. These results, at a $0.25 \mathrm{mM}$ concentration of drug substrate, were in general agreement with previous studies carried out at much higher substrate concentrations $[17,18,29]$. The substrate concentration used in the present study, $0.25 \mathrm{mM}$, was chosen to be reflective of plasma CPA and IFA concentrations in cancer patients, which can range from 0.1 to $0.5 \mathrm{mM}$, particularly in high-dose therapy studies $[2,14,30]$. The enzymatic rates for cDNA-expressed CYPs in the 4-hydroxylation reaction reported in this study were several-fold higher than those reported previously [15]; this is most likely due to the use of long incubation times $(60 \mathrm{~min})$ in the earlier studies, required to obtain sufficient product for accurate analysis.

\section{Role of CYP3A4 in Microsomal CPA N-Dechloroethylation}

The present identification of CYP3A4 as the principal enzyme responsible for CPA $N$-dechloroethylation in human liver microsomes was determined by the RSF method and was verified experimentally using the CYP3A-specific inhibitor TAO. This finding is consistent with the inhibi- 


\section{A. CPA + Mab2B6}

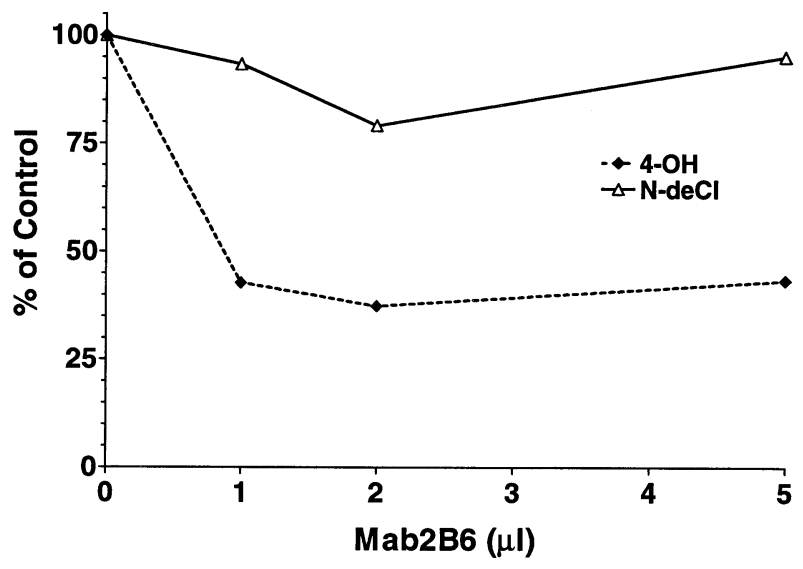

C. IFA + Mab2B6

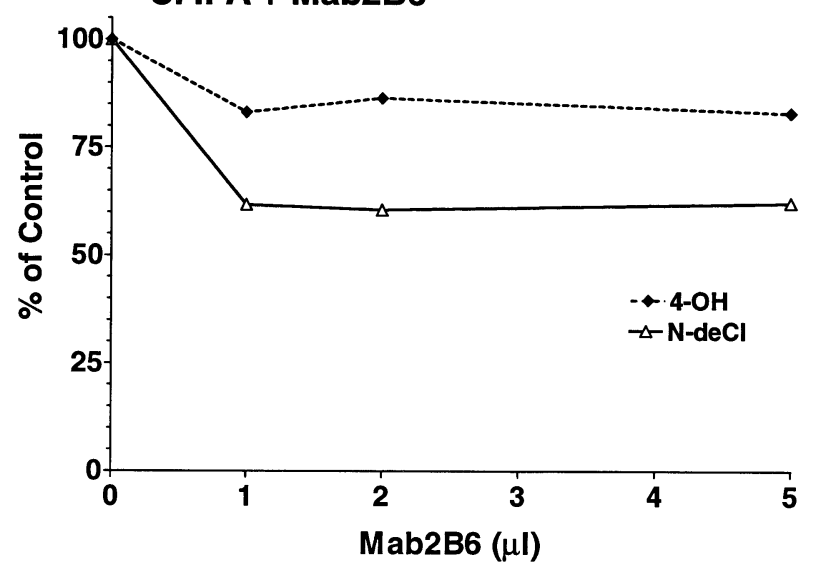

B. CPA + TAO

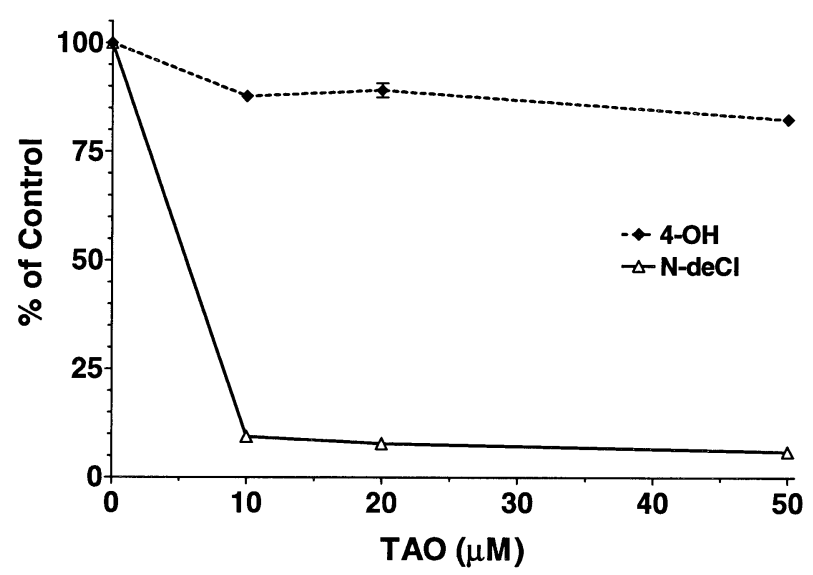

D. IFA + TAO

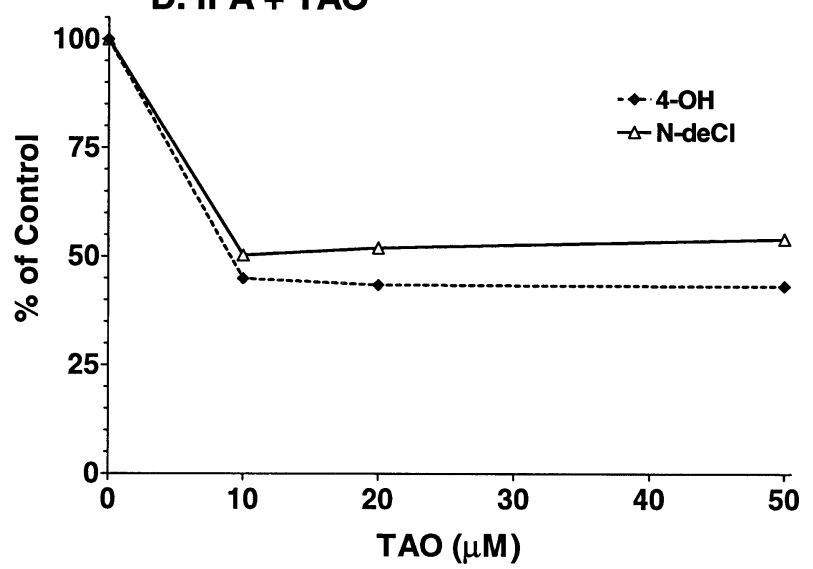

FIG. 4. Mab2B6 and TAO inhibition of CPA and IFA metabolism catalyzed by pooled human liver microsome H161. Inhibition assays were carried out as detailed in Materials and Methods. The stock concentration of Mab2B6 was $10 \mathrm{mg} / \mathrm{mL}$. Each data point represents the mean of duplicate determinations. Data are presented as a percentage of uninhibited activities determined in parallel in control incubations. The absolute activities in control incubations (100\% values) were: CPA 4-OH, $0.216 \mathrm{nmol} / \mathrm{min} / \mathrm{mg} \mathrm{protein}$; CPA $\mathrm{N}$-deCl, $0.048 \mathrm{nmol} / \mathrm{min} / \mathrm{mg}$ protein; IFA $4-\mathrm{OH}, 0.125 \mathrm{nmol} / \mathrm{min} / \mathrm{mg}$ protein; and IFA N-deCl, $0.120 \mathrm{nmol} / \mathrm{min} / \mathrm{mg} \mathrm{protein}$.

tion of liver microsomal CPA N-dechloroethylation by the CYP3A inhibitor ketoconazole [31]. By contrast, CYP3A4 is not a major enzymatic catalyst of microsomal CPA 4-hydroxylation, which is primarily catalyzed by CYP2B6 and by several other P450 enzymes, such as CYP2A6 and CYP2C enzymes [15]. Consequently, CYP3A-specific inhibitors, such as the macrolide antibiotic TAO, may be

TABLE 4. Inhibition of IFA N-dechloroethylation by Mab2B6 and TAO

\begin{tabular}{lccccc}
\hline & \multicolumn{2}{c}{ CYP2B6 } & & \multicolumn{2}{c}{ CYP3A4 } \\
\cline { 2 - 3 } \cline { 5 - 6 } HLM* & Experimental $\dagger$ & Predicted & & Experimental $\dagger$ & Predicted $\neq$ \\
\hline H161 & 44.5 & 36.8 & & 60.6 & 61.0 \\
H042 & 45.3 & 39.5 & & 59.6 & 59.4 \\
HLS2 & 74.8 & 58.2 & & 31.8 & 38.8 \\
HLS25 & 29.6 & 9.8 & & 80.2 & 87.5
\end{tabular}

*Human microsomes were those shown in Table 1.

$†$ Percentage of inhibition based on inhibitory effects of Mab2B6 (CYP2B6) or $\mathrm{TAO}$ (CYP3A4) in experiments similar to those shown in Fig. 4C and 4D.

†Predicted value based on RSF calculation as shown in Table 3. used to achieve a selective inhibition of liver microsomal CPA N-dechloroethylation, with little impact on CPA 4-hydroxylation. Support for this possibility is provided by

TABLE 5. Enzyme kinetics: CPA and IFA 4-hydroxylation and $\mathrm{N}$-dechloroethylation

\begin{tabular}{clccc}
\hline CYP & $\begin{array}{c}\text { Enzyme } \\
\text { reaction }\end{array}$ & $\begin{array}{c}K_{m} \\
(\mathrm{mM})\end{array}$ & $\begin{array}{c}V_{\max } \\
(\mathrm{nmol} / \mathrm{min} / \mathrm{nmol} \text { P450) }\end{array}$ & $V_{\text {max }} / K_{m}$ \\
\hline 2B66 & CPA 4-OH & 1.89 & 100.4 & 53.1 \\
& CPA N-deCl & & $<0.02$ & \\
& IFA 4-OH & 1.88 & 10.1 & 5.4 \\
& IFA N-deCl & 1.58 & 42.2 & 26.7 \\
3A4 & CPA 4-OH & 1.46 & 25.5 & 17.5 \\
& CPA N-deCl & 1.58 & 79.4 & 50.3 \\
& IFA 4-OH & 1.33 & 56.9 & 42.8 \\
& IFA N-deCl & 1.14 & 54.6 & 47.9
\end{tabular}

The parameters of enzyme kinetics were determined from Eadie-Hofstee plots using EnzymeKinetics software. Calculations based on other enzyme kinetics plots such as Lineweaver-Burke, Hanes-Woolf, Johansen-Lumry, direct linear plot, and nonlinear-regression analysis gave similar values, with variations $\leq 5 \%$. 
the finding that TAO completely inhibits CPA N-dechloroethylation in rat liver microsomes [24] and in human liver microsomes (Fig. 4) without any effect on CPA 4-hydroxylation. Moreover, in a rat pharmacokinetic study, TAO treatment in vivo completely suppressed CPA Ndechloroethylation while slightly increasing 4-hydroxylation [32]. However, CPA N-dechloroethylation in cancer patients only accounts for $\sim 10 \%$ of total CPA metabolism. Consequently, even if complete suppression of the $\mathrm{N}$ dechloroethylation pathway were to be achieved, the antitumor activity of CPA is not likely to be altered significantly. This supposition is supported by tumor excision and tumor growth delay studies carried out in the TAO-treated rat model [32].

\section{Role of CYP2B6 and CYP3A4 in Liver Microsomal IFA $\mathrm{N}$-Dechloroethylation}

Both CYP3A4 and CYP2B6 were found to contribute significantly to human liver microsomal IFA $\mathrm{N}$-dechloroethylation, as judged by the RSF method and by the use of P450 form-selective inhibitors. Because CYP3A4 also contributes significantly to IFA 4-hydroxylation [15, 18], it is unlikely that a selective inhibition of hepatic IFA $N$ dechloroethylation in vivo can be achieved using CYP3A4specific inhibitors such as TAO. Indeed, TAO inhibited IFA 4-hydroxylation and $\mathrm{N}$-dechloroethylation to the same extent in human liver microsomes (Fig. 4). In an earlier study [26], it was reported that CYP2B6 does not play a role in human liver microsomal IFA $N$-dechloroethylation based on the failure of 7-ethoxycoumarin to inhibit IFA $\mathrm{N}$-dechloroethylation, and it was concluded that IFA $\mathrm{N}$-dechloroethylation is catalyzed exclusively by CYP3A4. However, 7-ethoxycoumarin is not a CYP2B6-specific inhibitor, and the failure of such inhibition does not provide a reliable basis for this conclusion. In fact, TAO inhibited IFA $N$-dechloroethylation in human liver microsomes by only $\sim 70-80 \%$ in that study [26], consistent with our conclusion that other P450 enzymes (i.e. CYP2B6) are involved. CYP2B6 catalyzed $\sim 27 \%$ of human liver microsomal IFA $N$-dechloroethylation in an average liver in our studies, and the CYP2B6 contribution was as high as $40-70 \%$ of the total activity in CYP2B6-rich human livers, such as livers HLS2 and H042 (Table 4). Other evidence supporting a significant role for CYP2B6 in IFA N-dechloroethylation includes our recent finding that CYP2B6 is the primary human liver enzyme forming $(S)$-2-dechloroethylIFA and (S)-3-dechloroethyl-IFA during the $N$-dechloroethylation reaction [29]. In cancer patients, these two metabolites account for about $30-40 \%$ of total $N$-dechloroethylated IFA metabolites [33], a value that is in good agreement with our present conclusion that CYP2B6 catalyzes an average of $\sim 27 \%$ of total IFA $N$-dechloroethylation in human liver microsomes. Rat CYP2B1, which is orthologous to human CYP2B6, is also an important catalyst of IFA $\mathrm{N}$-dechloroethylation [24].

The significant role of CYP2B6 in human liver IFA
$\mathrm{N}$-dechloroethylation established by these findings is somewhat surprising, given the low level of CYP2B6 protein in human liver, reported to be less than 1\% of total P450 [34]. This raises the question as to how CYP2B6 could be a major microsomal catalyst of this reaction, given that the rate of IFA $N$-dechloroethylation catalyzed by cDNA-expressed CYP2B6 is comparable to, but not much greater than, that of the much more abundant liver enzyme CYP3A4 (Fig. 2). First, it should be noted that the level of CYP2B6 expression in human liver microsomes is actually higher than the value originally reported by Shimada et al. [34]. For example, the level of CYP2B6 protein in the panel of human liver microsomes used in the present study was 0.7 to $7 \%$ of total P450 (mean value 3.6\%) based on quantitative data obtained by the supplier using a CYP2B6-specific monoclonal antibody. Two other recent studies [35, 36] also demonstrate that the protein level of CYP2B6 in human livers is substantially higher than that initially reported [34], although the absolute protein levels showed great interindividual variation ( 0.3 to $74 \mathrm{pmol}$ CYP2B6/mg protein) $[35,36]$. Second, two other human liver microsomal activities specifically associated with CYP2B6, (S)-mephenytoin $\mathrm{N}$-demethylase and 7-ethoxy-4-trifluoromethylcoumarin $\mathrm{O}$-deethylase, are also significantly higher than the activities that would be expected based on the liver CYP2B6 protein content. For example, the measured CYP2B6 O-deethylase activity in human liver microsome samples HLS2, HLS8, HLS9, and HLS25 is 452, 36, 340, and $55 \mathrm{pmol} /$ $\mathrm{min} / \mathrm{mg}$ protein [36], whereas activity values directly calculated on the basis of the measured CYP2B6 protein contents of these liver microsomes and the specific O-deethylase activity of cDNA-expressed CYP2B6 [36] are up to 5to 7-fold lower (data not shown). A similar phenomenon was observed for CYP2B6-dependent microsomal IFA Ndechloroethylase activity in the present study. The reason for the much higher measured than predicted human liver microsomal CYP2B6 catalytic activity is not known at present. Conceivably, CYP2B6-dependent liver microsomal activities could be enhanced by an unknown substance in human liver via an allosteric activation mechanism [35], or perhaps the polyclonal and monoclonal antibodies used to measure CYP2B6 protein levels significantly underestimate the CYP2B6 content of human liver microsomes. Further investigation is required to clarify this point.

\section{Pharmacological Approaches to Minimize Conversion of IFA to CA}

Based on the more complete understanding of the P450 enzyme profiles for liver microsomal IFA metabolism provided by this study, several approaches to minimizing CA formation in IFA-treated patients can be considered. As noted above, CYP3A4 contributes significantly to both IFA $N$-dechloroethylation and IFA 4-hydroxylation in human liver microsomes, and thus it is likely to be difficult to selectively inhibit IFA $\mathrm{N}$-dechloroethylation in vivo using CYP3A4-specific inhibitors. Nevertheless, treatment 
of rats with the CYP3A inducer dexamethasone increased IFA 4-hydroxylation ( $\sim 3.5$-fold increase) without changing the $N$-dechloroethylation, thereby decreasing the percentage of IFA $N$-dechloroethylation from $36 \%$ of total IFA metabolism in untreated rat liver microsomes to $14 \%$ in dexamethasone-induced rat liver microsomes [24, 27]. The underlying mechanism for this selective decrease in IFA $\mathrm{N}$-dechloroethylation in the rat model is not clear, but this observation could provide a basis for minimizing CA formation in cancer patients. Another approach suggested by the present study is based on the selective inhibition of CYP2B6-mediated IFA N-dechloroethylation. Since CYP2B6 catalyzes an important fraction of IFA $\mathrm{N}$-dechloroethylation (but not IFA 4-hydroxylation) in human liver tissue, CYP2B6-selective inhibitors, such as orphenadrine and metyrapone, may be used to selectively inhibit the $N$ dechloroethylation pathway. This approach may be particularly useful for patients with CYP2B6-rich livers, but would not be effective in individuals with low CYP2B6 levels. When combined with the use of (R)-IFA in place of racemic IFA [29, 37], this approach could provide the opportunity to minimize CA-related toxicities and enhance therapeutic effects. Further preclinical and clinical studies will be required to evaluate the potential utility of these approaches to inhibition of IFA $N$-dechloroethylation.

The present study focused on the metabolism of IFA and CPA mediated by liver P450 enzymes. However, intestinal P450s may contribute significantly to IFA $\mathrm{N}$-dechloroethylation, as suggested by the fact that IFA undergoes a greater degree of $\mathrm{N}$-dechloroethylation during oral administration than following i.v. infusion [38]. In vitro studies have demonstrated that rat intestinal microsomes metabolize $>$ $90 \%$ of IFA via $\mathrm{N}$-dechloroethylation and that 3 -dechloroethyl-IFA is the major metabolite [39]. Only $\sim 47 \%$ of IFA was metabolized via $N$-dechloroethylation by rat hepatic microsomes [39]. Further characterization of intestinal P450 involved in IFA $N$-dechloroethylation could facilitate approaches to minimize the intestinal $\mathrm{N}$-dechloroethylation that may accompany the oral administration of IFA.

This work was supported, in part, by NIH Grant CA49248 (to D. J. W.).

\section{References}

1. Fleming RA, An overview of cyclophosphamide and ifosfamide pharmacology. Pharmacotherapy 17: 146S-154S, 1997.

2. Sladek NE, Metabolism and pharmacokinetic behavior of cyclophosphamide and related oxazaphosphorines. In: Anticancer Drugs: Reactive Metabolism and Interactions (Ed. Powers G), pp. 79-156. Pergamon Press, Oxford, 1994.

3. Dechant KL, Brogden RN, Pilkington $T$ and Faulds $D$, Ifosfamide/mesna: A review of its antineoplastic activity, pharmacokinetic properties and therapeutic efficacy in cancer. Drugs 42: 428-467, 1991.

4. Springate JE, Ifosfamide metabolite chloroacetaldehyde causes renal dysfunction in vivo. J Appl Toxicol 17: 75-79, 1997.

5. Pohl J, Stekar J and Hilgard P, Chloroacetaldehyde and its contribution to urotoxicity during treatment with cyclophosphamide or ifosfamide. An experimental study. Arzneimittelforschung 39: 704-705, 1989.

6. Zamlauski-Tucker MJ, Morris ME and Springate JE, Ifosfamide metabolite chloroacetaldehyde causes Fanconi syndrome in the perfused rat kidney. Toxicol Appl Pharmacol 129: 170-175, 1994.

7. Sood C and O'Brien PJ, 2-Chloroacetaldehyde-induced cerebral glutathione depletion and neurotoxicity. Br J Cancer 74(Suppl 27): S287-S293, 1996.

8. Thigpen $\mathrm{T}$, Ifosphamide-induced central nervous system toxicity. Gynecol Oncol 42: 191-192, 1991.

9. Kaijser GP, Korst A, Beijnen JH, Bult A and Underberg WJ, The analysis of ifosfamide and its metabolites. Anticancer Res 13: 1311-1324, 1993.

10. Kaijser GP, Beijnen JH, Bult A and Underberg WJ, Ifosfamide metabolism and pharmacokinetics. Anticancer Res 14: 517-531, 1994.

11. Alarcon RA, Fluorometric determination of acrolein and related compounds with m-aminophenol. Anal Chem 40: 1704-1708, 1968.

12. Bohnenstengel F, Eichelbaum M, Golbs E and Kroemer HK, High-performance liquid chromatographic determination of acrolein as a marker for cyclophosphamide bioactivation in human liver microsomes. J Chromatogr B Biomed Sci Appl 692: 163-168, 1997.

13. Kaijser GP, Ter Riet PG, de Kraker J, Bult A, Beijnen JH and Underberg WJ, Determination of 4-hydroxyifosfamide in biological matrices by high-performance liquid chromatography. J Pharm Biomed Anal 15: 773-781, 1997.

14. Wright JE, Tretyakov O, Ayash LJ, Elias A, Rosowsky A and Frei E III, Analysis of 4-hydroxycyclophosphamide in human blood. Anal Biochem 224: 154-158, 1995.

15. Roy P, Yu LJ, Crespi CL and Waxman DJ, Development of a substrate-activity based approach to identify the major human liver P450 catalysts of cyclophosphamide and ifosfamide activation based on cDNA-expressed activities and liver microsomal P450 profiles. Drug Metab Dispos 27: 655-666, 1999.

16. Clarke L and Waxman DJ, Oxidative metabolism of cyclophosphamide: Identification of the hepatic monooxygenase catalysts of drug activation. Cancer Res 49: 2344-2350, 1989.

17. Chang TK, Yu L, Goldstein JA and Waxman DJ, Identification of the polymorphically expressed CYP2C19 and the wild-type CYP2C9-ILE359 allele as low- $K_{m}$ catalysts of cyclophosphamide and ifosfamide activation. Pharmacogenetics 7: 211-221, 1997.

18. Chang TKH, Weber GF, Crespi CL and Waxman DJ, Differential activation of cyclophosphamide and ifosphamide by cytochromes P-450 2B and $3 \mathrm{~A}$ in human liver microsomes. Cancer Res 53: 5629-5637, 1993.

19. Ren S, Yang JS, Kalhorn TF and Slattery JT, Oxidation of cyclophosphamide to 4-hydroxycyclophosphamide and deschloroethylcyclophosphamide in human liver microsomes. Cancer Res 57: 4229-4235, 1997.

20. Weber GF and Waxman DJ, Activation of the anti-cancer drug ifosphamide by rat liver microsomal P450 enzymes. Biochem Pharmacol 45: 1685-1694, 1993.

21. Kaijser GP, Beijnen JH, Bult A, Wiese G, de Kraker J, Keizer $\mathrm{HJ}$ and Underberg WJ, Gas chromatographic determination of 2- and 3-dechloroethylifosfamide in plasma and urine. J Chromatogr 583: 175-182, 1992.

22. Ruzicka JA and Ruenitz PC, Derivatization-liquid chromatographic assay of chloroacetaldehyde in biological samples. J Chromatogr 518: 385-389, 1990.

23. Kaijser GP, Beijnen JH, Jeunink EL, Bult A, Keizer HJ, de Kraker J and Underberg WJ, Determination of chloroacetal- 
dehyde, a metabolite of oxazaphosphorine cytostatic drugs, in plasma. J Chromatogr 614: 253-259, 1993.

24. Yu L and Waxman DJ, Role of cytochrome P450 in oxazaphosphorine metabolism. Deactivation via $\mathrm{N}$-dechloroethylation and activation via 4-hydroxylation catalyzed by distinct subsets of rat liver cytochromes P450. Drug Metab Dispos 24: 1254-1262, 1996.

25. Ruzicka JA and Ruenitz PC, Cytochrome P-450-mediated $\mathrm{N}$-dechloroethylation of cyclophosphamide and ifosfamide in the rat. Drug Metab Dispos 20: 770-772, 1992.

26. Walker D, Flinois J-P, Monkman SC, Beloc C, Boddy AV, Cholerton S, Daly AK, Lind MJ, Pearson ADJ, Beaune PH and Idle JR, Identification of the major human hepatic cytochrome $\mathrm{P} 450$ involved in activation and $\mathrm{N}$-dechloroethylation of ifosfamide. Biochem Pharmacol 47: 11571163, 1994.

27. Huang $Z$ and Waxman DJ, HPLC-fluorescent method to determine chloroacetaldehyde, a neurotoxic metabolite of the anti-cancer drug ifosfamide, in plasma and in liver microsomal incubations. Analyt Biochem 273: 117-125, 1999.

28. Yang TJ, Krausz KW, Shou M, Yang SK, Buters JT, Gonzalez FJ and Gelboin HV, Inhibitory monoclonal antibody to human cytochrome P450 2B6. Biochem Pharmacol 55: 16331640, 1998.

29. Roy P, Tretyakov O, Wright J and Waxman DJ, Stereoselective metabolism of ifosfamide by human P450s 3A4 and 2B6. Favorable metabolic properties of R-enantiomer. Drug Metab Dispos 27: 1309-1318, 1999.

30. Kurowski V and Wagner T, Comparative pharmacokinetics of ifosfamide, 4-hydroxyifosfamide, chloroacetaldehyde, and 2 and 3-dechloroethylifosfamide in patients on fractionated intravenous ifosfamide therapy. Cancer Chemother Pharmacol 33: 36-42, 1993.

31. Bohnenstengel F, Hofmann U, Eichelbaum M and Kroemer $\mathrm{HK}$, Characterization of the cytochrome P450 involved in side-chain oxidation of cyclophosphamide in humans. Eur J Clin Pharmacol 51: 297-301, 1996.

32. Yu LJ, Drewes P, Gustafsson K, Brain EGC, Hecht JED and Waxman DJ, In vivo modulation of alternative pathways of P450-catalyzed cyclophosphamide metabolism: Impact on pharmacokinetics and antitumor activity. J Pharmacol Exp Ther 288: 928-937, 1999.

33. Granvil CP, Ducharme J, Leyland-Jones B, Trudeau M and Wainer IW, Stereoselective pharmacokinetics of ifosfamide and its 2- and 3-N-dechloroethylated metabolites in female cancer patients. Cancer Chemother Pharmacol 37: 451-456, 1996.

34. Shimada T, Yamazaki H, Mimura M, Inui Y and Guengerich $\mathrm{FP}$, Interindividual variations in human liver cytochrome P-450 enzymes involved in the oxidation of drugs, carcinogens and toxic chemicals: Studies with liver microsomes of 30 Japanese and 30 Caucasians. J Pharmacol Exp Ther 270: 414-423, 1994.

35. Ekins S, Vandenbranden M, Ring BJ, Gillespie JS, Yang TJ, Gelboin HV and Wrighton SA, Further characterization of the expression in liver and catalytic activity of CYP2B6. J Pharmacol Exp Ther 286: 1253-1259, 1998.

36. Code EL, Crespi CL, Penman BW, Gonzalez FJ, Chang TK and Waxman DJ, Human cytochrome P4502B6, Interindividual hepatic expression, substrate specificity, and role in procarcinogen activation. Drug Metab Dispos 25: 985-993, 1997.

37. Wainer IW, Granvil CP, Wang T and Batist G, Efficacy and toxicity of ifosfamide stereoisomers in an in vivo rat mammary carcinoma model. Cancer Res 54: 4393-4397, 1994.

38. Kurowski V, Cerny T, Kupfer A and Wagner T, Metabolism and pharmacokinetics of oral and intravenous ifosfamide. $J$ Cancer Res Clin Oncol 117: S148-S153, 1991.

39. Lu $\mathrm{H}$ and Chan KK, Differential in vitro hepatic and intestinal metabolism of ifosfamide in the rat. Xenobiotica 28: 779-784, 1998. 